\title{
Decolonizando el currículo. Una experiencia con cursos de lectura y escritura académicas*
}

\section{Decolonising the curriculum. An experience with academic reading and writing courses}

DAVID L. GARCÍA LEÓN**

JAVIER E. GARCÍA LEÓN***

Este artículo presenta algunos de los resultados de un proyecto aplicado a los cursos nivelatorios de lectoescritura de la Universidad Nacional de Colombia, sede Bogotá, durante los periodos académicos 2012-1 a 2013-1. Analiza el programa syllabus de los cursos con base en la investigación-acción participativa como enfoque metodológico y en los aportes teóricos del giro decolonial, en particular, el concepto de colonialidad del lenguaje. Los resultados revelan que el género argumental era la tipología discursiva primordial en la literacidad académica; se desconocían las prácticas culturales y lingüísticas de los estudiantes y se reproducía una mirada eurocéntrica y colonial. También, identifican una mirada formalista y remedial de la lectoescritura, pues los aspectos sintácticos y gramaticales desplazan elementos funcionales y comunicativos; por lo tanto, fue necesario decolonizar el programa del curso para vincular los saberes y modos de comunicación de los estudiantes.

This article presents the results of a research project on the reading and writing of Spanish language-level courses at the National University of Colombia (Bogotá) from 2012 to 2013. The article outlines the analysis of course syllabus from a Participatory Action Research methodology and from the contributions of the decolonial turn through the concept of language coloniality. Research results indicated that the courses prioritized the argumentative genre while ignoring the cultural and linguistic practices of the students which reproduced a colonial Eurocentric view on academic reading and writing. Additionally, the syllabus emphasised on the syntactic and grammatical aspects of language literacy which ignored its functional and communicative elements, resulting in a formalistic and corrective view of the language. Based on the research findings, the course syllabus was decolonized as a measure to link the linguistic and cultural practices of the (afro and indigenous) students.
Palabras clave:

literacidad crítica, lectura y escritura académicas, programa académico, educación terciaria, decolonialidad

\section{Keywords:}

critical literacy, academic reading and writing, academic programme, tertiary education, decoloniality

Recibido: 8 de agosto de 2018. | Aceptado para su publicación: 24 de enero de 2019. Recuperado de: https://sinectica.iteso.mx/index.php/SINECTICA/article/view/886 DOI: $10.31391 /$ S2007-7033(2019)0052-004

\footnotetext{
*Ambos autores contribuyeron equitativamente en la elaboración de este trabajo.

** Doctor en Estudios Hispánicos por la University of Ottawa. Profesor asistente del Departamento de Lenguas de la Pontificia Universidad Javeriana, Bogotá. Líneas de investigación: análisis del discurso, estudios latinoamericanos y de género, escritura y lectura académica, y enseñanza de lenguas y sociolingüística. Correo electrónico: Davidleogarcial@gmail.com/https://orcid.org/0000-0003-2360-5603

*** Doctor en Estudios Hispánicos por la University of Ottawa. Instructor de la Universidad Nacional Autónoma de México/Canadá. Líneas de investigación: análisis del discurso, estudios latinoamericanos y de género, escritura y lectura académica, enseñanza de lenguas y sociolingüística. Correo electrónico: Jgarc056@uottawa.ca/https:// orcid.org/0000-0003-3477-3346
} 


\section{INTRODUCCIÓN}

$\mathrm{E}$

ste artículo presenta los resultados de un proyecto investigativo de aula en torno a las implicaciones de la elaboración de un nuevo programa para los cursos nivelatorios de lectura y escritura en la educación superior, en particular aquellos ofrecidos a estudiantes de la Universidad Nacional de Colombia (UNAL). El análisis comprende los periodos académicos 2012-1 a 2013-1 en la sede Bogotá. El proyecto de aula es parte de una investigación más amplia titulada "Sentipensantes: leer y escribir desde y para la pluralidad en la universidad", cuyo objetivo central fue formular y desarrollar, con un grupo de estudiantes y profesores participantes en los cursos nivelatorios de lectoescritura (CNLE) de la UNAL, proyectos de lectoescritura en el aula orientados a fomentar el reconocimiento de la pluralidad en la vida académica universitaria. Sentipensantes fue una propuesta ganadora de la convocatoria de investigación Orlando Fals Borda, en 2012, de la Facultad de Ciencias Humanas de la UNAL, y fue elaborada y dirigida por las profesoras Gloria Mora y Doris Santos, adscritas a esta misma institución.

En este artículo presentamos, primer lugar, la metodología del estudio y, en seguida, una descripción de los cursos nivelatorios (sus orígenes, propósitos y población) y caracterización de los conceptos de literacidad académica (Carlino, 2003; Cassany, 2003) y currículo (Grundy, 1998). Luego, analizamos el programa de esos cursos desde la perspectiva de la investigación-acción participativa (IAP) (Freire, 1967; Fals-Borda \& Mora-Osejo, 2003) y los aportes teóricos del giro decolonial (Walsh, 2010b, 2015; Castro-Gómez, 2005), en especial el concepto de colonialidad del lenguaje (Veronelli, 2015). Por último, abordamos el proceso de construcción de un nuevo programa institucional que surgió de la puesta en práctica de un proyecto de investigación-acción entre los profesores y estudiantes de los cursos mencionados y cuyo objetivo final fue decolonizar las concepciones que, en el ámbito universitario, se tienen sobre la lectura y la escritura.

Con este trabajo buscamos contribuir al reconocimiento de la pluralidad en la construcción del currículo educativo, específicamente en el programa como guía de navegación de las actividades que se desarrollan en las aulas universitarias. Desde esta perspectiva, entonces, educar en y hacia la pluralidad y desde la decolonialidad implica construir dicha guía de manera colaborativa; esto es, incluir en su elaboración la voz de docentes y estudiantes, en especial la de aquellos alumnos que la epistemología colonial ha silenciado.

Este texto se presenta como una herramienta base para futuros y actuales docentes de lectoescritura a nivel universitario con miras a reflexionar en torno a las prácticas de construcción de programas académicos con enfoque decolonial. A la par, busca contribuir, en términos de Mignolo (2005), a la desobediencia epistémica al proponer otras formas de entender la lectura y la escritura en la universidad.

\section{Metodología}

Desde 2009, la UNAL decidió implementar los CNLE para los estudiantes con bajos niveles en el componente de compresión de lectura de la prueba de admisión a la universidad. Estos cursos fueron concebidos como una forma de "nivelar" a los que presentaban mayores dificultades para comprender textos de carácter académico. 
A estos cursos se vinculan los estudiantes del Programa de Admisión Especial, que busca brindar acceso a la educación superior a jóvenes pertenecientes a las comunidades indígenas y afrodescendientes del país. A la par, incluye a los mejores bachilleres de todo el país, en especial de regiones pobres.

Cada semestre, alrededor de 300 estudiantes de diferentes carreras toman dichos cursos en su primer semestre académico al ser clasificados por la Dirección Nacional de Admisiones de la UNAL. Estos cursos, además, son coordinados por el Departamento de Lingüística y enseñados, en su mayoría, por becarios asistentes docentes como parte de sus tareas. Con base en esta figura, los autores de este artículo nos vinculamos a los cursos durante los periodos 2012-1 y el 2013-1.

A inicios del primer semestre de 2013, los cursos nivelatorios formaron parte del proyecto de investigación "Sentipensantes: leer y escribir desde y para la pluralidad en la universidad", cuyo objetivo fue el desarrollo de prácticas de lectura y escritura que fomentaran la pluralidad y su reconocimiento en la vida académica. Esto significa que se buscaba convertir los cursos en un espacio donde la producción académica ayudara a mejorar las competencias en la lectura y escritura de los estudiantes desde una concepción social y práctica de estas; es decir, como competencias para la vida: "Capacidades -los conocimientos, las habilidades, los valores, las actitudes(necesarias) para enfrentar exitosamente los desafíos de la vida (privada, profesional y social) y las situaciones excepcionales, y también para continuar aprendiendo" (Atorresi, 2005, p. 2).

Asimismo, se intentaba que dichos cursos fueran un espacio donde la diversidad de prácticas culturales y de producción de conocimiento se reconociera. Aunque no se optó directamente por un enfoque interseccional, el proyecto investigativo de aula también pretendía reflexionar acerca de la concurrencia de diversas formas interconectadas de subordinación-opresión (Denis, 2008), en específico de tipo racial y lingüístico. Durante el análisis de los programas, se evidencia cómo estas impactan la construcción y concepción que los estudiantes poseen y desarrollan de la lectura y escritura académicas.

Específicamente, el proyecto investigativo de aula en torno a las implicaciones de la elaboración de un nuevo programa para los CNLE se llevó a cabo a partir de diferentes etapas. En primer lugar, organizamos discusiones con los estudiantes de los CNLE en las aulas ( 10 cursos en total por semestre, lo que equivale a alrededor de 300 estudiantes). Las discusiones iniciales se centraron en comprender los aspectos sociopolíticos y culturales de la lectura y la escritura en el ámbito académico y en el contexto colombiano. Pusimos de relieve las prácticas de escritura y lectura que desarrollan las comunidades minorizadas en el país, indígenas y afrocolombianas, dado que varios de los estudiantes de estos grupos pertenecían a este sector.

Posteriormente, llevamos a cabo discusiones entre los docentes de los cursos (cuatro personas), la coordinadora de estos, y la investigadora principal del proyecto Sentipensantes. Durante las quince discusiones entre profesores y coordinadores, presentamos avances del nuevo programa y abordamos sus implicaciones con una mirada pluralista, decolonial e incluyente. Estas discusiones también estuvieron alimentadas con datos de corte etnográfico que cada docente elaboró a partir de su trabajo en las aulas. Cada profesor, de manera autónoma, recogía información en torno al proceso de acompañamiento a los textos que sus estudiantes producían durante el curso.

García / García. Decolonizando el currículo. Una experiencia con cursos de lectura y escritura académicas Sinéctica 52 www.sinectica.iteso.mx 
Finalmente, luego de las sesiones de discusión, presentamos el nuevo programa de los cursos. Optamos por el uso de la IAP porque busca disminuir la injusticia en la sociedad, promover la participación de los miembros de comunidades para solucionar sus propios problemas e incrementar el grado de control que los individuos poseen sobre aspectos relevantes de sus vidas (Balcazar 2003); en este caso, la escritura y lectura académicas en el ámbito universitario.

\section{AnÁlisis. Pensando el CURRículo}

En este apartado analizamos el currículo desde diferentes postulados teóricos y de prácticas que, como investigadores, becarios asistentes y docentes de los cursos, llevamos a cabo. En otras palabras, nuestro análisis pone en diálogo dos perspectivas: los desarrollos teóricos sobre el currículo, la lectoescritura académica y la interculturalidad junto con la IAP y los aportes del giro decolonial.

Es importante recordar que generar conocimiento a partir de la IAP significa acompañar los procesos de transformación social y política que genere conocimiento crítico para transformar las condiciones cotidianas de vida de los individuos con los que se investiga (Esteva, 2017). Por ello, la producción de saber involucra transformar situaciones históricas concretas; esto es, trabajar de la mano con los sujetos participantes en la investigación y escuchar su sentipensar con el objetivo de modificar su situación contextual específica (Fals, 1987).

De este modo, la primera reflexión que surgió como producto de la IAP giro en torno a la forma en que se habían denominado los CNLE y los efectos que esto generaba en los alumnos a nivel motivacional y afectivo. Como su nombre lo indica, estos cursos fueron pensados como espacios académicos correctivos, pues el objetivo principal de la asignatura era superar las aparentes carencias que los estudiantes poseían en el campo de la lectoescritura. Dicha concepción producía algunos efectos negativos en el sentipensar de los estudiantes. Durante las clases concebidas con base en los postulados de la IAP, muchos alumnos manifestaron su desmotivación frente al curso. En su opinión, al estar matriculados en los CNLE, la comunidad académica los concebía como "rezagados", lo que generaba una jerarquía de estudiantes en el campus. Sumado a esto, varios estudiantes sostenían que era injusta su pertenencia a ese espacio, ya que consideraban que sus competencias en lectoescritura eran superiores a las que se reflejaban en la evaluación inicial.

Esta situación evidencia de manera clara el legado de la colonización en la academia colombiana; es decir, refleja una colonialidad epistémica, ontológica y corpopolítica en tanto que se jerarquizan los sujetos y sus prácticas lingüísticas por medio de concepciones eurocéntricas de entender la comunicación. La idea de que las competencias comunicativas deben ser corregidas o niveladas parte de una concepción colonial del lenguaje en la que "se conecta la lengua, la gramática, la civilización y la escritura alfabética con el conocimiento, y [se] naturalizan estas características y atributos como lenguaje 'en sentido pleno'” (Veronelli, 2015, p. 48). Este fenómeno condujo a que, como investigadores-docentes, debiéramos repensar dos elementos esenciales del currículo de este curso: su conceptualización y la forma en que se concibe la lectura y la escritura académicas en la educación terciaria. 
En cuanto al primer aspecto, empezamos a problematizar el término currículo y preferimos abordarlo desde la propuesta de la pedagogía crítica, en especial la desarrollada por Grundy (1998, p. 22) y Freire (1997). Aunque partimos de la propuesta de estos dos autores, reconocemos que la pedagogía crítica, y en particular la latinoamericana, posee un carácter multifacético que, como señala Cabaluz-Ducasse (2016), se caracteriza de tres elementos. En primer lugar, "afluentes teórico-políticos de tipo democrático popular, socialista, marxista, libertario y cristiano, entre otros” (p. 76). Segundo, esta pedagogía emana "del pensamiento pedagógico contrahegemónico desplegado por pedagogos tales como Paulo Freire, Moacir Gadotti, Darcy Ribeiro, Gaudencio Frigotto, Demerval Saviani, Tomaz Tadeu da Silva (Brasil); Carlos Cullen, Carlos Alberto Torres..." (p. 76).

Por último, la pedagogía crítica latinoamericana parte de

la multiplicidad de experiencias de organización y lucha emanadas desde el movimiento popular latinoamericano, en los saberes constituidos por las organizaciones y los movimientos sociales, en la profunda memoria social del continente, en las identidades fragmentadas de nuestros pueblos y en las inagotables formas de creatividad y asociatividad popular (Cabaluz-Ducasse, 2016, p. 76).

De este modo, entendemos la pedagogía desde una mirada decolonial, siguiendo la propuesta de Mignolo y Walsh (2018), pues es una disciplina cuya metodología se basa en las realidades, subjetividades, historias y luchas de las personas con quienes se trabaja.

Por lo anterior, no concebimos el currículo como un objeto a priori; esto es, como un marco de lineamientos que se conciben de manera previa a la práctica de la enseñanza y el aprendizaje, sino como una práctica cultural que se va construyendo constantemente con los sujetos involucrados en esta y atendiendo las realidades sociales en las que estos se encuentran inmersos (García y García, 2012). Esto significa que tanto docentes como estudiantes problematizamos los imaginarios que poseíamos sobre los cursos nivelatorios y, a partir de esa reflexión, empezamos a crear una mirada más coherente de la realidad que enmarcaba el curso. Decidimos que era necesario cuestionar la mirada que se tiene sobre la lectura y la escritura en la universidad y discutir el imaginario que se había construido sobre los cursos nivelatorios, espacios donde se suplen las “falencias" de la educación básica y media.

Sin embargo, antes de aproximarnos a esa reflexión, tuvimos que acercarnos a los procesos históricos que han generado la estructura actual de las instituciones universitarias. Para ello fue necesario posicionar las universidades latinoamericanas como

estructuradas alrededor de la transmisión de los conocimientos científicos, tecnológicos y humanísticos que fueron estandarizados en las universidades occidentales después de una gran reforma epistémica que los distanció del modo integrado de saberes, característico de las instituciones europeas desde el Renacimiento hasta el inicio de la Ilustración (Carvalho y Flórez, 2014 p. 133).

Este rechazo al modo integrado de saberes implicó que se dejaran por fuera los conocimientos provenientes de comunidades afro, indígenas y campesinas; este hecho se refleja en los actuales programas de enseñanza y en las aulas. De esta manera, surgió la necesidad de cuestionar la perspectiva que se asumía en el curso frente a los procesos históricos que han acompañado el desarrollo de las universidades colombianas, y se ha reconocido que en ellas se asume una forma eurocéntrica de concebir la educación.

García / García. Decolonizando el currículo. Una experiencia con cursos de lectura y escritura académicas Sinéctica 52 www.sinectica.iteso. $m x$ 
Luego de esta contextualización, las primeras sesiones con los estudiantes se orientaron a establecer qué perspectiva curricular había sido heredada, cuál era el enfoque que en realidad deberían asumir los cursos de lectura y escritura, y cómo estas asignaturas deberían ser reestructuradas. En otras palabras, pusimos en práctica uno de los principios básicos de la IAP: la praxis pedagógica como acto problematizador de develamiento y desnaturalización de la realidad opresora a fin de avanzar en la prefiguración de nuevas relaciones sociales menos dominantes (Gadotti, 1996).

Para esa problematización, no solo organizamos sesiones de discusión; también propusimos lecturas que nos permitieran identificar los intereses o marcos ideológicos desde los cuales habían sido concebidos los cursos. Esto nos condujo a entender que la concepción de los cursos nivelatorios era sesgada y no contribuía a entender el problema de la lectura y la escritura académicas en su totalidad. Por ello, fue necesario reflexionar sobre la lectura y la escritura no como tareas elementales que se alcanzan de manera mecánica y que no deben tocarse en el ámbito universitario al ser un asunto de la educación secundaria.

Esta visión limitada nos llevó a indagar sobre lo que implica leer y escribir en la educación terciaria y a identificar, siguiendo los postulados de Carlino (2003), que estas prácticas en el ámbito universitario poseen unas características propias y, por consiguiente, muy diferentes a aquellas de la educación básica y media. Así, la lectura y la escritura a nivel universitario

son necesarias para aprender a pensar críticamente dentro de los marcos conceptuales de cada disciplina, b) [para] ingresar en la cultura escrita de cualquier dominio de conocimiento exige dominar sus prácticas discursivas características, c) [y para] producir e interpretar textos especializados, según los modos académicos [además] implica capacidades aún en formación no alcanzables espontáneamente (Carlino, 2003, p. 416).

De esta manera, haber cuestionado la concepción sobre lectura y escritura en la universidad nos permitió entender que estos espacios no deben concebirse como algo remedial, sino que las propias prácticas y características de la academia exigen el desarrollo de habilidades de lectura y escritura específicas que se encuentran muy vinculadas a cada una de las disciplinas y que deben ser usadas de manera constante, y hacer que se mejoren y desarrollen a medida que el estudiante se sumerge en los discursos propios de su área de conocimiento.

Luego de reflexionar en torno a la definición y caracterización que se poseía de los CNLE, emergió otro elemento primordial: comprender la necesidad de una perspectiva emancipadora decolonial en la construcción del currículo y la educación universitaria en general. Esto quiere decir que los estudiantes y los docentes nos dimos cuenta de la importancia de problematizar la realidad y la forma en que los discursos nos la presentan.

El sencillo ejercicio de cuestionar el objetivo del curso en el que participábamos nos llevó a asumir una mirada crítica sobre las prácticas educativas, especialmente en aquellas que la universidad impone. El discurso en torno a lo remedial y del déficit fue problematizado y, por consiguiente, desnaturalizado, pues los estudiantes empezaron a concebirse como sujetos que contribuyen a la construcción de su quehacer en la universidad, dado que son ellos quienes participan en la elaboración del currículo. Por lo tanto, pudimos construir un currículo emancipador en dos niveles: reflexivo y práctico. Para Grundy (1998), este tipo de currículo posee dos niveles: 
Ante todo, un nivel de la consciencia, los sujetos que participan en la experiencia educativa llegarán a saber teóricamente y en términos de su propia existencia cuándo las proposiciones representan perspectivas deformadas del mundo y cuándo representan regularidades invariantes de existencia. En el nivel de la práctica, el curriculum emancipador implicará a los participantes en el encuentro educativo, tanto profesor como alumno, en una acción que trate de cambiar las estructuras en las que se produce el aprendizaje y que limitan la libertad de modos con frecuencia desconocidos (p. 39).

Por lo tanto, la constante reflexión acerca de los discursos que nos interpelan desde lo educativo nos condujo a repensar el papel que tenemos en la construcción de nuestro propio aprendizaje y a ejercer control sobre este. Así, los estudiantes comenzaron a evidenciar que pertenecer a los cursos nivelatorios no se debe concebir como algo negativo; además, les permitió entender que su papel en la construcción de ese espacio es vital, pues su participación mejora las prácticas de aprendizaje y de enseñanza, al asumir un papel más autónomo y menos bancario, si seguimos los términos utilizados por Freire en cuanto a su concepción de las prácticas educativas (1997).

Vale la pena mencionar que estos ejercicios reflexivos llevaron a una mejor comprensión de lo que significa la actual estructuración de universidades colombianas; en especial, el hecho de que forman parte de un legado histórico de la colonialidad, en los que se excluyen, estigmatizan y posicionan como imperantes diversos saberes e individuos. La escritura y lectura académicas no son ajenas a estos procesos y descolonizar sus programas implica una reflexión sobre el origen de las universidades como espacios donde se perpetúa, pero también se puede subvertir la colonialidad.

Además, una pedagogía en clave decolonial implica construir un currículo emancipatorio que, por una parte, ubique, resignifique y vincule las experiencias y los saberes de aquellos que intervienen en los proyectos con la finalidad de proporcionar una transformación crítica de la realidad social (Díaz, 2010 p. 225). Es importante mencionar que las lenguas indígenas colombianas, que muchos de los estudiantes de los CNLE poseen, han sido históricamente excluidas de la escritura académica. Colombia ha establecido unas políticas lingüísticas que van muchas veces en su detrimento (García y García, 2013), ya que se fundamentan en los legados históricos de la colonialidad. Al respecto, Sarzuri-Lima (2012) ha manifestado que con la colonización se propagó la idea de que las lenguas indígenas carecen de escritura, lo que propició que

se las ubique en la base de la clasificación lingüística; los indios al "carecer" de escritura sólo poseen dialectos, entonces son personas analfabetas, sin letra y palabra. Esto lleva a una conclusión sencilla: los indios, al estar privados de una escritura, se encuentran privados de la razón. Esta reducción mecánica de la escritura (alfabética y con base textual en el papel) invisibiliza la naturaleza amplia y plural de la escritura (p. 65).

Para nosotros fue esencial debatir esa invisibilización como lo mostramos en el siguiente apartado.

\section{REVISANDO Y DISCUTIENDO EL PROGRAMA}

Durante el periodo comprendido entre 2012-1 y 2013-1, utilizamos y discutimos el programa del curso, que es caracterizado y analizado a continuación (ver anexos 1 y 2). En primer lugar, este destacaba el desarrollo de la competencia argumentativa. Los autores del programa consideraban que este elemento habría de ser el eje fundamental sobre el cual se debería guiar el curso: "Esta asignatura tiene como 
propósito contribuir al desarrollo de las competencias de lectura y escritura de textos argumentativos tanto orales como escritos" (ver anexo 1). Esta característica condujo a que se presentaran dos fenómenos que fueron manifestados por los estudiantes en las diferentes sesiones de reflexión y discusión desarrolladas en el curso. Primero, el resalte en la argumentación era contradictorio, hasta cierto punto, con la necesidad de mejorar las habilidades de lectura y escritura propias de cada disciplina. Esto sucedía en la medida que no todos los campos tienen como textos prototípicos aquellos que se pueden ubicar en la tipología argumentativa, pues el modo explicativo es más recurrente en algunas áreas del conocimiento.

Este hecho generó que durante el curso se construyera, desde la praxis pedagógica, un programa paralelo. Con los estudiantes de los cursos, establecimos que era necesario dar cabida a otras tipologías textuales. Así, a lo largo del semestre se discutieron textos narrativos, descriptivos y explicativos. Al mismo tiempo y como proyecto transversal, se elaboró un texto argumentativo tipo ensayo. Esto permitió vincular las necesidades particulares de los estudiantes, en especial de aquellos en cuyas disciplinas los modos explicativo y descriptivo eran más relevantes.

Cabe destacar que el hacer hincapié en la argumentación no se dejó del todo de lado por dos razones. En primer lugar, porque la argumentación permea los discursos de la cotidianidad y, en segundo, porque el discurso académico, independientemente de la disciplina en que se manifieste, es un ejercicio de poder en el cual la argumentación está presente (Dib, 2007). La argumentación se convirtió en un elemento central debido a la coyuntura política que pasaba la UNAL. En los periodos académicos aquí descritos, los trabajadores de esta institución entraron en huelga como mecanismo para demandar mejores condiciones laborales. Estas protestas impactaron las actividades académicas y fueron propicias para discutir sobre las problemáticas sociales que afectaban y afectan a los miembros de la comunidad universitaria; esto mejoró las habilidades argumentativas.

Asimismo, fue necesario cuestionar algunos discursos que en un primer nivel se presentan como neutros por su "naturaleza" científica. Los estudiantes, por ejemplo, asociaban ciencia con neutralidad y objetividad. Incluimos textos académicos de disciplinas como las matemáticas, la física y la agronomía debido a que varios estudiantes pertenecían a estas áreas; sin embargo, discutimos cómo el discurso científico, a través de diversas estrategias lingüísticas, se muestra como totalmente objetivo y verdadero, y oculta, algunas veces, su relación con las esferas sociales, económicas, políticas y culturales. Gracias a estas discusiones, los alumnos mejoraron sus competencias en comprensión de textos académicos y en lectura crítica. Además, entendieron que

... los discursos no son neutros ni reflejan objetiva e inocuamente la realidad, porque siempre son el resultado de la producción lingüística de un enunciador, en un lugar y un momento concretos, que inevitablemente tiene unas intenciones, unos puntos de vista y un conocimiento del mundo determinados (que son particulares e irrepetibles, puesto que no existen seres humanos ni situaciones comunicativas iguales). Esto es cierto para cualquier texto y lo sería también, por ejemplo, para los artículos de investigación científica: por mucha objetividad que se busque, cada investigación se contextualiza en un momento histórico y en un lugar concreto del devenir de la humanidad (Cassany, 2003 p. 4).

Por otro lado, debemos resaltar que no se buscaba cambiar totalmente el programa del curso. Aunque este presentaba algunas limitaciones, no se podía desconocer el 
trabajo ganado a lo largo de los semestres anteriores, ya que, desde la perspectiva curricular asumida y descrita en el apartado anterior, el currículo es una práctica que se construye con los sujetos involucrados, se da la voz a todos y se intenta, a partir de sus aportes, construir caminos de aprendizaje y enseñanza que conduzcan a mejorar las habilidades disciplinares y a construir relaciones de poder menos verticales.

El ejercicio de investigación-acción, además, condujo a la inclusión de conocimientos todavía no validados en el ámbito académico y dentro del currículo del curso. Como mencionamos, los estudiantes de los cursos nivelatorios poseen diversas prácticas sociales y culturales, pues, a modo de ejemplo, un número importante de ellos proviene de grupos que han sido marginados socialmente en Colombia, las comunidades indígenas y afrodescendientes. Los saberes de estos alumnos y su forma de transmitirlos estaban determinados por la colonialidad del lenguaje o por la relación raza-lengua desde un enfoque filosófico, político e ideológico eurocéntrico que "permite a la imaginación colonial presuponer a los colonializados como seres menos que humanos, expresiva y lingüísticamente" (Veronelli, 2015, p. 48).

Este efecto de la colonialidad del lenguaje se manifestó en forma de rechazo, ya que algunos de los estudiantes cuestionaron el fuerte acento que el programa curricular le asignaba a la redacción de ensayos académicos. Para las comunidades a las que pertenecía una gran parte de los estudiantes, la narración y la oralidad juegan un papel central en la construcción y divulgación de saberes. Estas no solo les permiten construir formas particulares de entender la realidad, sino también llevar a cabo prácticas argumentativas que no necesariamente están mediadas por el ensayo académico tradicional. Recuérdese que la escritura y la oralidad están atravesadas por la colonialidad lingüística y epistémica. La primera cobra un papel más relevante que la segunda, pues se presenta como universal y se desconocen sus legados orales; sin embargo, "la escritura, como la propia base de la comunicación, debe incluir todos los ejemplos de templetes con los cuales la voz interactúa para producir sentido" (Garcés, 2007 p. 227), sentido que desde el mundo andino "incluiría todas las prácticas textuales: tejido, canciones, bailes, etc." (Garcés, 2007 p. 228).

Además, el ensayo se instituía en una forma eurocéntrica de construcción y representación de los saberes, al jerarquizar los modos del discurso y establecerse como el único modo válido para la representación del conocimiento científico; en otras palabras, esta tipología textual partía de una política lingüística colonial. Este fenómeno va de la mano de lo que el grupo modernidad-colonialidad (Castro-Gómez, $2005,2007)$ ha denominado la colonialidad del saber y la hybris del punto cero, en donde los conocimientos de las comunidades colonizadas son presentados como doxa ('opinión'), en tanto que aquellos desarrollados desde Europa se presentan como científicos y carentes de intereses particulares. A este respecto, Castro-Gómez (2007) sostiene:

Observados desde el punto cero, los conocimientos que vienen ligados a saberes ancestrales, o a tradiciones culturales lejanas o exóticas, son vistos como doxa, es decir, como un obstáculo epistemológico que debe ser superado. Solamente son legítimos aquellos conocimientos que cumplen con las características metodológicas y epistémicas definidas a partir del mismo punto cero. Los demás conocimientos, desplegados históricamente por la humanidad durante milenios, son vistos como anecdóticos, superficiales, folclóricos, mitológicos, "pre-científicos" y, en cualquier caso, como pertenecientes al pasado de Occidente. Este colonialismo epistémico de la ciencia occidental no es en absoluto gratuito. La hybris 
del punto cero se forma, precisamente, en el momento en que Europa inicia su expansión colonial por el mundo, en los siglos XVI y XVII, acompañando así las pretensiones imperialistas de Occidente (p. 88).

Como se evidencia, la dominación europea que se ejerció en distintos ámbitos logró jerarquizar los saberes y discursos sobre las otras culturas con el propósito de considerar los conocimientos europeos como los únicamente válidos. Este se constituyó como objetivo científico y verdadero y se abandonaron opciones alternativas endógenas que responden a las necesidades culturales, históricas y políticas propias de las comunidades tradicionales latinoamericanas (Fals-Borda \& Mora-Osejo, 2003).

En consecuencia, es vital asumir una pedagogía emancipadora en clave decolonial que descentre las formas canónicas de producir y organizar el conocimiento en las prácticas educativas actuales a nivel universitario. Esto supone, como afirma Cristhian James Díaz, "someter a reflexión crítica su funcionamiento habitual, y en especial, los principios pedagógicos propios de la modernidad que le informan y le caracterizan" (2010 p. 228). Por ello, discutir, en un curso de lectoescritura académica, el papel del ensayo como eje central del programa es someter a reflexión crítica las pautas que informan la construcción del currículo actual en relación con procesos históricos coloniales precedentes.

Partiendo de esta mirada, la IAP implementada tuvo como propósito incluir otras formas de producir, transmitir, socializar y difundir el conocimiento al interior de los CNLE. Por esta razón, reestructuramos el programa académico de estos cursos. En esta etapa de la investigación, buscamos dar mayor espacio a la tipología narrativa, pues esta forma discursiva permite entender otras realidades, además de cuestionar el conocimiento considerado como válido o científico. Durante los periodos académicos mencionados, los estudiantes produjeron diversas narraciones que refutaban la forma en que se elabora el conocimiento en las universidades y cómo este se jerarquizaba a través del lenguaje, pues el discurso narrativo y los contenidos que este transmite son representados en estas instituciones, muchas veces, como anecdóticos y folclóricos, en otros términos, no científicos.

Asimismo, durante el semestre 2013-1, trabajamos con los estudiantes en la construcción de un ensayo. Sin embargo, como éramos conscientes de las discusiones en torno a la validación del conocimiento científico dentro de la universidad, optamos por que cada estudiante escogiera el tema de su interés, para que, de esta manera, pudiera comunicar sus propias experiencias sin desconocer las exigencias típicas que este tipo de texto posee.

Así, desarrollamos las competencias en escritura que esta clase de discurso exige y permitimos a los estudiantes tener un papel más protagónico en el aula. Esta fue una modificación importante en tanto que en semestres anteriores era el grupo de docentes quienes seleccionaban los temas para el ensayo. Esto generaba que los estudiantes se sintieran obligados a escribir sobre temas que no necesariamente les interesaban, lo que contradecía los principios de la IAP, en la que todos los participantes tienen un rol relevante en la toma de decisiones. Por lo tanto, otorgamos validez a otras formas de construir conocimiento y también hicimos hincapié en la autonomía del estudiante, tal como la propone Freire (1997). 
Para este autor, el conocimiento debe ser significativo y esto implica un rol protagónico por parte del estudiante. Este debe participar en la realización de las tareas de aprendizaje y cuestionar la realidad en la que se encuentra. Haber permitido a los estudiantes escoger el tema de los ensayos condujo a que muchos de ellos abordaran aspectos sociopolíticos de sus disciplinas. Temas como el racismo, la minería, la agricultura a gran escala, la eutanasia, la despenalización de las drogas, la autonomía universitaria, entre otros, fueron propuestos, desarrollados y discutidos por los estudiantes.

De este modo, se consolidó la competencia escrita desde un enfoque en el que esta no solo es necesaria para el desempeño universitario, sino que, como afirman De la Peza, Rodríguez, Hernández y Rubio (2014), incide en la capacidad de las personas para tomar decisiones fundadas y desempeñarse con plenitud en la esfera pública. A esto se suma que muchos de estos textos fueron publicados en la revista Prima exagia, un espacio diseñado especialmente para que los estudiantes de los CNLE divulguen sus escritos. Esta revista fue creada desde el 2011 con la coordinación de la profesora Gloria Mora y es dirigida por el grupo de apoyo a los cursos nivelatorios, conformado por estudiantes del Departamento de Lingüística de la Universidad, con el apoyo financiero de la Dirección de Bienestar de la sede.

Otro aspecto importante que se modificó fue el objetivo central del curso. La concepción de que los CNLE eran asignaturas remediales estaba vinculada a la idea de que estos espacios eran, en resumen, cursos de gramática y sintaxis del español y que buscaban enmendar vacíos provenientes de la educación media; por ejemplo, al inicio del semestre y luego de hacer una lectura del programa junto con los estudiantes, estos consideraban que los ejercicios de puntuación y de sintaxis serían recurrentes. Estas concepciones las suscitó el lenguaje demasiado teórico con el que había sido escrito el programa; términos como sintaxis, semántica, pragmática, acto de habla, falacia, práctica discursiva, entre otros, generaban incomprensión y preocupación por parte de los estudiantes, quienes suponían que el curso sería un taller de sintaxis avanzada del español (ver anexo 1). Sin embargo, el propósito del curso era diferente; se trataba de mejorar las habilidades comunicativas, lo cual implica asumir una perspectiva más discursiva frente al uso del lenguaje y, por lo tanto, vincular la comunicación con los fenómenos sociales.

La situación descrita nos obligó a reestructurar el plan temático del curso durante las primeras sesiones. De esta manera, comenzamos el espacio académico con algunas reflexiones sobre la comunicación en vez de iniciar con el texto argumentativo como se proponía inicialmente. Este cambio fue bastante significativo para los estudiantes, pues pudieron abordar textos cotidianos, como la publicidad, y en los cuales se presentan rasgos argumentativos, y conectarlos con las realidades discursivas en las que viven. Por lo tanto, asumir una perspectiva más comunicativa y discursiva y menos estructuralista y textual les permitió adquirir herramientas de interpretación y producción de discursos que van mucho más allá de aquellas requeridas para la argumentación y el discurso académico, al vincular sus prácticas comunicativas con la universidad.

En este punto es importante cuestionar el uso de metalenguajes en la estructuración de los programas de las diferentes asignaturas. Sin duda, para participar dentro de cualquier comunidad académica, es necesario conocer en profundidad los conceptos de la disciplina estudiada. Sin embargo, al referirnos a la participación de los estudiantes en los cursos de lectura y escritura, este presupuesto debe abordarse con 
mayor cautela. Es indispensable potenciar las habilidades de comprensión y producción de discursos y no explicar, necesariamente, los fenómenos lingüísticos que constituyen los discursos. Al desatenderse las necesidades y prácticas comunicativas de los estudiantes, se les desmotiva y se reproduce la idea de que el estudio del lenguaje no implica un vínculo con los contextos sociales donde este se desarrolla. Esto niega los desarrollos propios de la lingüística y, sobre todo, la desliga de su papel social.

En nuestra opinión y como lo evidenciaron los estudiantes a lo largo del curso, mejorar la comunicación escrita y la interpretación discursiva debe servir para construir sujetos políticos capaces de participar con argumentos informados en las discusiones que se dan en las esferas social y política; es decir, el uso del lenguaje, al estar enmarcado en prácticas sociales, puede contribuir a mantener formas de dominación, pero también a su deconstrucción (Fairclough, 2003); hacia esto último se deben orientar los cursos de lectura y escritura, y abandonar una postura estructuralista y poco funcionalista.

\section{CONSTRUYENDO UN NUEVO PROGRAMA}

En este apartado explicamos el proceso de estructuración del nuevo programa del curso. Para este proceso, como se mencionó en la metodología, tuvimos en cuenta las voces de todas las personas involucradas en los CNLE durante los periodos académicos 2012-1, 2012-2 y 2013-1; esto significa que los estudiantes, docentes, miembros del grupo de apoyo a los cursos y la coordinadora contribuyeron, desde sus experiencias y reflexiones, a la elaboración del programa que presentamos a continuación, respetando los postulados centrales de la IAP.

A diferencia del curso anterior, los temas no fueran seleccionados solo por los docentes, pues buscábamos deslegitimar la figura del experto. Esta siempre ha estado asociada a una apropiación del conocimiento por parte del sujeto colonizador blanco y a las prácticas epistemológicas del norte global (Fairclough, 2008; Fals-Borda \& Mora-Osejo, 2003). Además, el objetivo era incorporar las voces y opiniones de los estudiantes y de los miembros del grupo de apoyo, pues, de lo contrario, se hubieran generado dos fenómenos. En primer lugar, perpetuaríamos una perspectiva limitada de los cursos nivelatorios y, por lo tanto, desconoceríamos los avances y saberes que estos dos actores han generado. En segundo, violaríamos los principios metodológicos sobre los que se fundamentaba esta IAP.

Inicialmente, en una serie de reuniones con los alumnos reflexionamos sobre las ventajas y problemáticas que poseía el programa y propusimos posibles mejoras. Esos encuentros se organizaron a lo largo de los periodos académicos con la intención de incluir las concepciones de todos los actores involucrados en el desarrollo del curso, sobre todo de los alumnos. Como cierre del semestre académico de 2013, decidimos, de manera grupal, replantear y reelaborar el programa de los CNLE. La primera modificación consistió en presentar, de modo explícito y claro, la perspectiva que se tiene sobre la lectura y la escritura académica (ver figura 1). 


\section{DESCRIPCIÓN}

Esta asignatura tiene como propósito contribuir al desarrollo de las competencias de lectura y escritura de diversos tipos de textos, especialmente aquellos que son parte de la vida académica universitaria. El curso es una oportunidad para mejorar los niveles de lectura y escritura; al mismo tiempo, busca hacer del paso de la educación media a la Universidad un proceso concatenado y con mejores resultados (Véase anexo 2)

Figura 1. Presentación de la perspectiva sobre la lectura y la escritura académica

Como se evidencia, ampliamos el espectro de discursos trabajados durante el semestre. Esto significa que, además de tener en cuenta el modo argumentativo, el curso permitiría mejorar la producción y comprensión de textos a nivel general. Esta modificación no cambiaba el enfoque del curso, pues los discursos académicos seguían estando presentes; sin embargo, permitía la entrada de otros tipos de textos y generaba que el programa fuera más coherente con la diversidad de estudiantes que lo cursan.

Asimismo, la concepción de lectura y escritura se modificó para que los estudiantes entendieran que estas dos actividades no son estáticas ni terminan en la educación media. Preferimos conceptualizar la lectoescritura como un proceso continuo en el cual cada etapa de formación (básica, media y universitaria) posee características propias que se interrelacionan con las demás. A la par, esto nos permitió superar la concepción limitada que se tenía sobre la lectoescritura. Recordemos que los CNLE se concibieron para suplir un déficit en la escritura académica, pues se consideraba que los estudiantes llegaban a la universidad con dificultades no superadas en la secundaria y con una concepción limitada de la alfabetización académica. Por lo tanto, pretendíamos que los futuros estudiantes entendieran que la lectura y la escritura a nivel universitario presentan desafíos particulares. Estas son un proceso inacabado que se practica constantemente al participar en la comunidad académica (ver figura 2)

\section{JUSTIFICACIÓN}

Este curso se hace necesario en tanto que uno de los propósitos centrales de la Universidad Nacional es la formación de profesionales integrales. En primer lugar, esto significa que los estudiantes deben estar en capacidad de leer y escribir apropiadamente los textos académicos característicos de su área profesional. Además, este curso contribuye a la formación integral en tanto que las competencias discursivas permean cualquier práctica social. Es decir, estas no solo están presentes en los ámbitos de la educación formal sino también en la cotidianidad. Por último, la importancia de este curso se basa en la idea de que la lectura y la escritura son procesos que nunca culminan, por ello su cualificación debe ser constante y procesual. Así, el curso ayuda a consolidar y mejorar los saberes aprendidos en la educación media y los potencializa como parte fundamental del paso a la Universidad.

Figura 2. Justificación del curso.

En tercer lugar, consideramos que era pertinente mantener la argumentación como punto de partida para el desarrollo de la lectura crítica. La argumentación no se limita al ámbito académico, sino que se expande al cotidiano y al profesional. De esta manera, asumimos una perspectiva mucho más funcional de la lengua y pusimos de relieve la formación de sujetos críticos que cuestionen las relaciones de poder contenidas en el lenguaje (ver figura 3).

El eje transversal que guía el desarrollo de la asignatura es la argumentación, pues se considera que la lectura y la escritura críticas son fundamentales para desempeñarse en contextos académicos, profesionales y cotidianos.

Figura 3. Argumentación.

García / García. Decolonizando el currículo. Una experiencia con cursos de lectura y escritura académicas Sinéctica 52 www.sinectica.iteso. $m x$ 
Las mejoras al programa incluyen los objetivos propios del curso, los intereses de los estudiantes y consideran la necesidad de formar sujetos críticos capaces de cuestionar y transformar las prácticas sociales en las que se desenvuelven.

En cuanto al plan temático, preferimos usar un lenguaje menos teórico y destacar los aspectos comunicativos y funcionales del uso del lenguaje. Abandonamos conceptos demasiado específicos de la lingüística y conceptualizamos la lectura y la escritura como prácticas sociales y no como prácticas puramente lingüísticas y cognitivas. Esta postura de ninguna manera significó desconocer los aspectos formales que subyacen a la producción e interpretación de textos; más bien ubicaba estas dos habilidades en espacios históricos, sociales y comunicativos concretos. Así, la postura crítica que asumimos frente al uso del lenguaje y el desarrollo de la competencia argumentativa quedó plasmada de forma más coherente en el programa académico.

A la par, el eje temático se estructuró de manera mucho más amplia si lo comparamos con el programa anterior: se pasó de una perspectiva muy estructuralista y específica del lenguaje a una mirada más global y práctica del uso de la lengua; por ejemplo, en vez de describir contenidos como "Generalidades gramaticales: nivel lexical, nivel morfosintáctico, puntuación, marcadores textuales" y "Oración y proposición”, estos fueron aglomerados en un solo enunciado: "La relación forma y contenido en los discursos escritos" (ver anexos 1 y 2). Esta modificación fue necesaria dado que es en la práctica con los estudiantes donde realmente los ejes temáticos toman vida.

En este sentido, son los docentes, junto con sus nuevos grupos, quienes ponen en práctica dichos temas y evalúan la relevancia y orden de los contenidos según las necesidades específicas de cada tipo de estudiantes. Como mencionamos, este cambio, a su vez, es coherente con la perspectiva curricular y metodológica asumida: una práctica cultural que se va construyendo constantemente con los actores involucrados en el proceso de enseñanza-aprendizaje.

Para cerrar este apartado, es importante señalar que, aunque las modificaciones al programa buscaban que este respondiera a los intereses y saberes de los estudiantes, no pretende convertirse en un documento estático de carácter aplicativo. Reiteramos que es necesaria su reestructuración constante teniendo en cuenta los intereses particulares de los estudiantes, sus necesidades académicas y los objetivos propios del curso. Esto supone entender la relevancia de incluir al estudiante y sus perspectivas en la construcción de cualquier currículo a fin de generar un giro educativo que permita la entrada del sujeto, sus valores, sensibilidades y voluntades por aprender y conocer (Zemelman, 2002), es decir, su historia personal y académica como elemento central en la emergencia de los procesos de enseñanza-aprendizaje.

\section{REFLEXIONES FINALES}

Esperamos que las reflexiones expuestas contribuyan a repensar la forma como los docentes construimos los programas y concebimos el currículo. Esto es relevante en la medida en que muchos de nosotros tendemos a elaborar los programas de los cursos sin consultar las opiniones e ideas que los estudiantes tienen sobre la asignatura; desconocemos los aportes que muchos de ellos pueden tener y reforzamos ideas erróneas y coloniales sobre las disciplinas y áreas que enseñamos. 
Ignorar las necesidades e intereses de nuestros estudiantes es silenciar sus voces y conocimientos. Este hecho contribuye a la consolidación de un monocentrismo y eurocentrismo académico. En este sentido, invitamos a pensar los espacios académicos como lugares interculturales donde se tejen todo tipo de relaciones (de poder) en torno a la construcción de conocimiento. Abogamos porque las universidades y sus aulas se conviertan en un sitio donde la interculturalidad crítica tenga cabida, como lo plantea Walsh (2010a): “... como proyecto político, social, ético y epistémico -de saberes y conocimientos-, que afirma la necesidad de cambiar no solo las relaciones, sino también las estructuras, condiciones y dispositivos de poder que mantienen la desigualdad, inferiorización, racialización y discriminación” (pp. 75-96).

Por último, somos conscientes de las limitaciones que este ejercicio de IAP posee, pues la decolonización de la escritura y lectura académicas involucra muchos más participantes, otras formas de consultar sus perspectivas sobre estos asuntos, otras prácticas pedagógicas en las que la construcción de los programas esté a cargo de la comunidad educativa y no solo del docente, y la puesta en práctica de proyectos de IAP más duraderos y de mayor envergadura. Sin embargo, esperamos que la experiencia narrada en estas páginas pueda abrir otros espacios de desobediencia epistémica y, así, contribuir a la decolonización del lenguaje.

De igual modo, este ejercicio investigativo pretende cuestionar y modificar las relaciones de dominación imperantes en el aula. Abogamos por otorgar mayor presencia a las voces, necesidades y formas de construir y transmitir el conocimiento que los estudiantes poseen, en especial las de aquellos que la colonialidad ha silenciado constantemente.

\section{REFERENCIAS BIBLIOGRÁFICAS}

Atorresi, Ana (2005). Competencias para la vida en las evaluaciones de lectura y escritura (SERCE-LLECE). Buenos Aires: Unesco/OREALC. Recuperado de http://ebookbrowse.com/competencias-vida-evaluaciones-lectura-escritura-atorresi-pdf-d316084667

Balcazar, Febricio (2003). Investigación acción participativa (IAP): aspectos conceptuales y dificultades de implementación. Fundamentos en Humanidades, vol. 4, núm. 1-2, pp. 59-77. Recuperado de http://www.redalyc.org/articulo.oa?id=18400804

Carlino, Paula (2003). Alfabetización académica: un cambio necesario, algunas alternativas posibles. Educere, vol. 6, núm. 20, pp. 409-420. Recuperado de http://www.saber.ula.ve/handle/123456789/19736

Cassany, Daniel (2003). Aproximaciones a la lectura crítica: teoría, ejemplos y reflexiones. Tarbiya, vol. 32, pp. 113-132.

Castro-Gómez, Santiago (2007). Decolonizar la universidad. La hybris del punto cero y el diálogo de saberes. En Santiago Castro-Gómez y Ramón Grosfoguel (eds.). El giro decolonial. Reflexiones para una diversidad epistémica más allá del capitalismo global (pp. 79-92). Bogotá: Siglo del Hombre Editores/ Universidad Central/Instituto de Estudios Sociales Contemporáneos y Pontificia Universidad Javeriana/Instituto Pensar.

Castro-Gómez, Santiago (2005). La poscolonialidad explicada a los niños. Popayán: Universidad del Cauca. 
Cabaluz-Ducasse, Jorge Fabián (2016). Pedagogías críticas latinoamericanas y filosofía de la liberación: potencialidades de un diálogo teórico-político. Educación y Educadores, vol. 19, núm. 1, pp. 67-88. Recuperado de http://educacionyeducadores.unisabana.edu.co/index.php/eye/article/view/5061

De Carvalho, José Jorge y Flórez Flórez, Juliana (2014). Encuentro de saberes: proyecto para decolonizar el conocimiento universitario eurocéntrico. Nómadas, núm. 41, pp. 131-147. Recuperado de http://www.redalyc.org/ articulo.oa?id=105133774009

De la Peza, Ma. del Carmen, Rodríguez Torres, Lilia R., Hernández Unzueta, Iliria y Rubio, Ricardo (2014). Evaluación de competencias de lectoescritura en alumnos de primer ingreso a la Universidad Autónoma Metropolitana-Xochimilco. Argumentos, vol. 27, núm. 74, pp. 117-148. Recuperado de https:// www.redalyc.org/articulo.oa?id=59532371006

Denis, Ann (2008). Intersectional analysis: A contribution of feminism to sociology. International Sociology, vol. 23, núm. 5, pp. 677-694.

Díaz, Cristhian James (2010). Hacia una pedagogía en clave decolonial: entre aperturas, búsquedas y posibilidades. Tabula Rasa, núm. 13, pp. 217-233. Recuperado de http://www.redalyc.org/articulo.oa?id=39617525009

Dib, Jimena (2007). ¿Cómo leer la dimensión argumentativa de los textos académicos? En Irene Klein (ed.). El taller del escritor universitario (pp. 62-73). Buenos Aires: Prometeo.

Esteva, Gustavo (2017). La construcción del saber histórico de lucha. En Catherine Walsh (ed.). Pedagogías decoloniales: prácticas insurgentes de resistir, (re)existir y (re)vivir, tomo II (pp. 77-103). Quito: Abya Yala.

Fairclough, Norman (2008). El análisis crítico del discurso y la mercantilización del discurso público: las universidades. Discurso y Sociedad, vol. 2, núm. 1, pp. 170-185.

Fairclough, Norman (2003). Analysing discourse. Textual analysis for social research. EUA: Routledge.

Fals Borda, Orlando (1987). Ciencia propia y colonialismo intelectual. Los nuevos rumbos. Bogotá: Carlos Valencia Editores.

Fals-Borda, Orlando \& Mora-Osejo, Luis (2003). Context and diffusion of knowledge. A critique of Eurocentrism. Action Research, vol. 1, núm. 1, pp. 2937. https://doi.org/10.1177/14767503030011003

Freire, Paulo (1997). Pedagogía de la autonomía saberes necesarios para la práctica educativa (11 ed.). México: Siglo XXI.

Freire, Paulo (1967). Pedagogía del oprimido. Bogotá: América Latina.

Gadotti, Moacir (1996). Pedagogía de la praxis. Buenos Aires: Miño y Dávila Editores.

Garcés, Fernando (2007). Las políticas del conocimiento y la colonialidad lingüística y epistémica. En Castro-Gómez Santiago y Ramón Grosfoguel (eds.). El giro decolonial. Reflexiones para una diversidad epistémica más allá del capitalismo global (pp. 217-242). Bogotá: Siglo del Hombre Editores/Universidad Central/Instituto de Estudios Sociales Contemporáneos y Pontificia Universidad Javeriana/Instituto Pensar.

García León, Javier y García León, David (2013). Educar en la posmodernidad: hacia una concepción pluralista y política. Educere, núm. 56, pp. 27-32. Recuperado de http://www.saber.ula.ve/bitstream/handle/123456789/38105/ articulo3.pdf? sequence=1\&isAllowed=y 
García León, Javier y García León, David (2012). Políticas lingüísticas en Colombia, tensiones entre políticas para lenguas mayoritarias y lenguas minoritarias. Boletín de Filología, núm. 47, pp. 47-70. http://dx.doi.org/10.4067/ S0718-93032012000200002

Grundy, Shirley (1998). Producto o praxis del curriculum. España: Ediciones Morata, SL.

Mignolo, Walter (2005). ). Cambiando las éticas y las políticas del conocimiento: lógica de la colonialidad y postcolonialidad imperial. Tabula Rasa, núm. 3, pp. 47-72.

Mignolo, Walter \& Walsh, Catherine (2018). On decoloniality: Concepts, analytics, praxis. Durham: Duke University Press. https://doi-org.proxy.bib.uottawa. $\mathrm{ca} / 10.1215 / 9780822371779$

Sarzuri-Lima, Marcelo (2012). De la palabra al texto: colonialidad lingüística y luchas interculturales. Integra Educativa, vol. 1, núm. 4, pp. 59-76. Recuperado de http://www.scielo.org.bo/scielo.php?script=sci_arttext\&pid=S199740432012000100003\&lng=es\&tlng=es.

Veronelli, Gabriela Alejandra (2015). Sobre la colonialidad del lenguaje. Universitas Humanística, núm. 81, pp. 33-58. https://doi.org/10.11144/Javeriana.uh81.scdl

Walsh, Catherine (2010a). Interculturalidad crítica y educación intercultural. En Jorge Viaña Jorge, Luis Tapia y Catherine Walsh (eds.). Construyendo interculturalidad crítica (pp. 75-96). Bolivia: Instituto Internacional de Integración del Convenio Andrés Bello.

Walsh, Catherine (2010b). Decolonial pedagogies walking and asking. Notes to Paulo Freire from AbyaYala. International Journal of Lifelong Education, vol. 34, núm. 1. Pp. 9-21. https://doi.org/10.1080/02601370.2014.991522

Zemelman, Hugo (2002). Necesidad de conciencia. Un modo de construir conocimiento. Barcelona: Anthropos. 


\section{Anexo 1. Programa inicial}

Curso nivelatorio en lectura y escritura

Información básica

Período académico: 2012-02

Asignatura: Curso nivelatorio en lectura y escritura Tipo: Nivelatorios

Código: 1000002 Grupo:

Intensidad horaria: 4 horas presenciales y 8 de trabajo autónomo

Horario: Créditos: 4

Profesor(a): Correo electrónico:

Horario de atención:

Oficina:

Edificio:

\section{Justificación}

Esta asignatura tiene como propósito contribuir al desarrollo de las competencias de lectura y escritura de textos argumentativos tanto orales como escritos. Para ello, se retoman principios teóricos provenientes de la sintaxis, la semántica, la pragmática y del análisis del discurso, así como principios metodológicos provenientes del campo de investigación en lectura y escritura. Esto ante la necesidad que tienen los estudiantes de cualquier carrera de la Universidad, de desarrollar sus competencias para leer y escribir los textos académicos propios de su área profesional.

\section{Objetivos}

El objetivo de este curso nivelatorio es cualificar la competencia argumentativa de los estudiantes, a nivel oral y escrito, por medio de la apropiación de estrategias de comprensión y expresión. Al finalizar el curso, los estudiantes estarán en capacidad de:

2.1. Identificar distintos modos discursivos presentes en un texto argumentativo.

2.2. Identificar la estructura argumentativa de un texto: tesis central o hipótesis, premisas, red de relaciones entre las premisas.

2.3. Emitir un juicio de valor en relación con la estructura identificada.

2.4. Identificar las distintas posturas que origina la argumentación contenida en el texto.

2.5. Construir textos que tengan una estructura argumentativa. 


\section{Plan temático}

3.1 Reflexiones sobre el lenguaje.

3.1.1. Generalidades: funciones del lenguaje, actos de habla, prácticas discursivas.

3.1.2. Posibilidades y límites del lenguaje escrito: oral-escrito; formal-informal.

3.1.3. Argumentación y persuasión.

3.1.4. Estructura global del texto argumentativo.

3.1.5. Modos discursivos en el texto argumentativo.

\subsubsection{Oración y proposición.}

3.1.7. Generalidades gramaticales: nivel lexical, nivel morfosintáctico, puntuación, marcadores textuales.

3.1.8. Algunos tipos textuales: la reseña, el informe.

3.2. Análisis de textos argumentativos (artículos científicos y ensayos).

3.2.1. Reconocimiento de la situación comunicativa.

3.2.2. Identificación de la situación problemática, del interrogante y del objeto de estudio.

3.2.3. Identificación de la estructura argumentativa: precisión de la tesis o conclusión, identificación de las premisas o argumentos explícitos e implícitos, red de relaciones.

3.2.4. Identificación de las relaciones argumentales (inferenciales) que se encuentran dentro del texto: inductivas, deductivas, falaciosas.

3.2.5. Análisis de la estructura formal del texto (título, formas de citación, notas, resumen, etc.).

3.2.6. Evaluación global del texto. Falacias.

\subsection{Construcción de textos argumentativos.}

3.3.1. Planeación/reflexión.

3.3.2. Delimitación del tema y formulación de la pregunta, búsqueda y procesamiento de la información.

3.3.3. Delimitación de la situación comunicativa.

3.3.4. Bosquejo de la estructura del texto.

3.3.5. Construcción de diferentes versiones del texto.

3.3.6. El proceso de revisión como actividad fundamental en la redacción: aspectos sintácticos, lógicos, semánticos, estilísticos, pragmáticos, ortográficos.

\section{Metodología}

4.1. El procedimiento metodológico básico para el desarrollo de la clase será el de taller, de suerte que los diferentes conceptos objeto de interiorización y aplicación por parte de los estudiantes se presentarán en la medida en que vayan surgiendo en la práctica de la lectura y la escritura.

4.2. El trabajo a lo largo del curso estará orientado hacia la elaboración de un 
ensayo. Esto supondrá realizar actividades para delimitar un tema concreto, recopilar información sobre dicho tema, jerarquizar tal información, hacer reflexiones a partir de las lecturas realizadas, elaborar textos parciales y un texto final de modo que quien lo lea lo comprenda.

4.3. Los estudiantes corregirán algunos escritos de sus compañeros. Para ello se harán explícitos y se comentarán los criterios de corrección a tener en cuenta.

4.4. Como parte de las actividades metodológicas y en congruencia con el principio de proporcionar contextos reales para la difusión de los textos de los estudiantes, se realizará el Octavo Encuentro de Estudiantes de los Cursos Nivelatorios de Lectura y Escritura el 28 de noviembre (por confirmar), el cual es obligatoria asistencia.

5. Actividades de evaluación

Elaboración de reseñas. 20\% Parcial. 20\%

Otras actividades y ejercicios. 20\% Examen final: Prueba escrita. 20\% Ensayo final. 20\% 


\title{
Anexo 2. Programa ReElaborado LUego de la IAP
}

\author{
Curso nivelatorio en lectura y escritura información básica
}

Periodo académico: 2013-II

Asignatura: Curso nivelatorio en lectura y escritura

Tipo: Nivelatorios

Código: 1000002 Grupo:

Intensidad horaria: 4 horas presenciales y 8 de trabajo autónomo

Horario: Créditos: 4

Profesor(a): Correo electrónico:

Horario de atención:

Oficina: Edificio:

\section{Descripción}

Esta asignatura tiene como propósito contribuir al desarrollo de las competencias de lectura y escritura de diversos tipos de textos, especialmente aquellos que son parte de la vida académica universitaria. El curso es una oportunidad para mejorar los niveles de lectura y escritura; al mismo tiempo, busca hacer del paso de la educación media a la Universidad un proceso concatenado y con mejores resultados. El eje transversal que guía el desarrollo de la asignatura es la argumentación, pues se considera que la lectura y la escritura críticas son fundamentales para desempeñarse en contextos académicos, profesionales y cotidianos. Por otra parte, el desarrollo de este curso se fundamenta en el trabajo colaborativo y autónomo. Por lo tanto, es esencial la relación entre las actividades realizadas en el aula con el docente (presencial), las asesorías brindadas por el grupo de apoyo a los estudiantes de lectoescritura, el trabajo en grupos (colaborativo) y el trabajo independiente (individual).

\section{Justificación}

Este curso se hace necesario en tanto que uno de los propósitos centrales de la Universidad Nacional es la formación de profesionales integrales. En primer lugar, esto significa que los estudiantes deben estar en capacidad de leer y escribir apropiadamente los textos académicos característicos de su área profesional. Además, este curso contribuye a la formación integral en tanto que las competencias discursivas permean cualquier práctica social. Es decir, estas no solo están presentes en los ámbitos de la educación formal sino también en la cotidianidad. Por último, la importancia de este curso se basa en la idea de que la lectura y la escritura son procesos que nunca culminan, por ello su cualificación debe ser constante y procesual. Así, el curso ayuda a consolidar y mejorar los saberes aprendidos en la educación media y los potencializa como parte fundamental del paso a la Universidad. 


\section{Objetivos}

Dado que el objetivo central de este curso es contribuir al desarrollo de las competencias de lectura y escritura de diversos textos, se espera que los estudiantes logren los siguientes objetivos al finalizar el curso:

3.1 Reflexionar sobre el papel de la lectura y la escritura como parte integral de la formación profesional.

3.2 Reconocer la argumentación como un eje transversal en las prácticas académicas, profesionales y cotidianas.

3.3 Reflexionar sobre los elementos involucrados en la comunicación y las prácticas discursivas.

3.4 Identificar los diversos tipos de textos propios de su formación profesional.

3.5 Reconocer las distintas posiciones y razones que subyacen a una producción discursiva.

3.6 Emitir un juicio crítico en relación con los textos leídos.

3.7 Producir discursos coherentes y apropiados a la intención y situación comunicativa.

3.8 Establecer los procesos cognitivos que subyacen a la producción y comprensión escrita.

3.9 Identificar las dificultades más comunes en la producción e interpretación discursiva y poner en práctica estrategias para solucionarlos.

Plan temático

Reflexiones en torno a la lectura y la escritura como prácticas sociales.

- Conceptualización sobre el lenguaje y la comunicación.

- Los géneros y las tipologías discursivas.

- La relación forma y contenido en los discursos escritos.

- Los procesos involucrados en la producción y comprensión de textos.

- La argumentación como práctica social y discursiva (análisis y construcción de textos argumentativos).

- Estrategias metacognitivas para mejorar la producción e interpretación discursiva.

\section{Metodología}

Los principios metodológicos que guían este curso son los estilos de aprendizaje entendidos como las diversas formas que tienen los estudiantes de adquirir el conocimiento y ponerlo en práctica. Además, dado que la escritura y la lectura son prácticas sociales, se considera que el aprendizaje colaborativo es esencial para alcanzar los objetivos del curso; por ello, se sugiere que el desarrollo del programa se dé por medio de talleres o proyectos donde los estudiantes, profesores y miembros del grupo de apoyo trabajen conjuntamente. De este modo, los diferentes conceptos 
objeto de interiorización y aplicación por parte de los estudiantes se trabajarán en la medida en que vayan surgiendo en la práctica de la lectura y la escritura y con ayuda de todos los miembros del curso.

Adicionalmente, se recomienda que las actividades que se realicen para abordar el plan temático partan del aprendizaje significativo; esto es, tener en cuenta los intereses y necesidades de los estudiantes. Por último, dichas actividades también deben fundamentarse en el enfoque comunicativo del aprendizaje de lenguas, en tanto que la producción y la interpretación del discurso son prácticas comunicativas reales. En congruencia con estos principios, se proponen actividades como: coproducción y co-evaluación de textos, exposiciones, lectura crítica de documentos, participación en discusiones, asistencia a tutorías, participación en el Encuentro de Estudiantes de los Cursos Nivelatorios de Lectura y Escritura, entre otras.

Para el desarrollo del curso, además de la bibliografía propuesta, se cuenta con recursos virtuales como el blog "Grupo de Apoyo a los Cursos Nivelatorios en Lectoescritura de la Universidad Nacional de Colombia”, donde se encuentran diversas herramientas para mejorar la escritura y la lectura. Además, se organizan, en la biblioteca central, algunas sesiones en relación con el manejo de bases de datos, catálogos y referencias.

\section{Actividades de evaluación}

Dentro del curso, la evaluación se concibe como un proceso. Por ello, más que valorar productos textuales finales, se busca evaluar los pasos y alcances que los estudiantes llevan a cabo para mejorar sus competencias en lectura y escritura. Se recomiendan, entre otras, las siguientes actividades como mecanismos de evaluación:

- Talleres, controles de lectura, ejercicios y otros.

- Parciales teóricos y aplicados.

- Elaboración de reseñas, resúmenes, informes.

- Portafolio de escritura.

- Elaboración de versiones preliminares de textos.

- Elaboración de ensayos, textos narrativos, explicativos, etc.

- Exposiciones grupales o individuales. 